\title{
Análises de Alguns Calcáreos Paranaenses
}

\author{
Alsedo Leprevost \\ Instituto de Biologia e Pesquisas Tecnológicas
}

\begin{abstract}
We may conclude that, in the analyses presented in the enclosed table, besides the natural existence in the same bearing, a great oscillation in every percentage:

1) the samples with quantity in $\mathrm{MgO}$ up to 3\%, represent 58,59\%, of the total analyses; from 3 to 5, 7,62\%; from 5 to $10,6,77 \%$; and above 10, 33,05\%.

2) with quantity insoluble up to 5\%, 54,23\%; from to 10, 16,10\%; and above 10, 27, $96 \%$.

3) of the analyses in which the dosage was made of the $\mathrm{MnO}, 47,27 \%$ gave negative result.

4) with the exception of the larger part of the white calcareous, every other one contain graphitic carbon.

5) the calcareous found in the Itaiacoca region, Ponta Grossa County, besides the mixture of magnesium silicate, there an $\mathrm{Mg}(\mathrm{OH})^{2}$ in variable quantities.

6) although some samples found in large bearing have presented good purity, it is necessary a complete rigorous study of the samples, according to the purpose, for there exist in them a large variety, mainly in $\mathrm{MgO}$.

7) due to the fact that the analyses were made with material largely send to this Instituto, the percentages figured in the above items refer only to the samples mentioned, and not an average in the State of Paraná..
\end{abstract}

Key Words: Calcareous; State of Paraná; analytical chemistry

\section{INTRODUÇÃO}

Grande é a aplicação dos calcáreos na indústria moderna, possuindo cada qual suas especificações técnicas próprias, motivo porque se torna complexa sua procura e seu beneficiamento.

Assim, por exemplo, a indústria do cimento exige baixo teor em magnésio e a da borracha não admite manganês.

Por sua vez, a da celulose, na preparação da lixívia de sulfito de cálcio pelo processo das torres, necessita baixos teores em magnésio e de insolúvel e isenção de carbono grafítico, ao passo que preparando-o pelo processo Franck estes inconvenientes desaparecem, podendo-se trabalhar até com cal de origem dolomítica.
Também a da cal, ora prefere calcáreo sem magnésio, ora admite-o em porcentagens elevadas. Em Mineralogia denomina-se de "calcáreo, ao carbonato de cálcio, compacto ou de gran fina, não cristalino, chamando-se aos cristalinos de mármores. São em geral de origem orgânica, conchas de moluscos, corais, etc., ou então são depósitos de precipitação" (Backheuser).

Naturalmente existem sempre, acompanhando o carbonato de cálcio, em maior ou menor proporção, outros carbonatos como os de magnésio, ferro, manganês e mais raramente de zinco, bário, etc. com a variação da quantidade destes, recebem os minerais novas denominações, como "dolomita", quando o teor em magnésio é

\footnotetext{
${ }^{\wp}$ Artigo publicado no Arquivos de Biologia e Tecnologia, v. 1, pp. 113-127, 1946.
} 
muito elevado, ou "anquerita", quando os teores em ferro e manganês atingem certos limites.

O carbonato de cálcio pode ainda apresentar-se cristalizado em romboedros, caso em que se denomina de calcita, ou em forma aparentemente hexagonal, chamando-se então aragonita.

Variável é também a quantidade e a qualidade do material com composição diversa da do calcáreo, que é retido durante a formação do mineral, dado na análise como insolúvel.

Apresentando este conjunto de análises de minerais diferentes, mas relacionados a uma mesma classe, generalizamos o termo, subordinando-os ao título de "calcáreos".

Os calcáreos do Estado do Paraná ocorrem principalmente na série Assunguí (= Série Minas), sendo sua composição muito variada, dentro de uma mesma jazida, predominando os dolomíticos e as dolomitas verdadeiras, aparecendo com alguma frequiência jazidas de calcita muito pura, porém em geral de pequena possança.

Além do elevado teor médio em magnésio, encerram apreciáveis valores em carbono grafítico e algumas vezes fortes porcentagens em insolúveis.

Os calcáreos até agora conhecidos em nosso Estado, ocorrem nas seguintes Séries: Assunguí, Passa Dois e Tubarão-Itararé.

Os da Série Assunguí, do algonquiano, nos Municípios de Paranaguá, Imbuial, Piraquara, Cerro Azul, Colombo, Campo Largo, Castro e Ponta Grossa, são principalmente industrializados para cal virgem e cal hidratada, estando em estudos, os de Cerro Azul, visando a fabricação de cimento; os de Imbuial e Castro foram já explorados como mármores.

Os de Tomazina pertencem à Série TubarãoItararé, do carbonífero superior, e os de Mallet à Série Passa Dois, do permiano superior.

As análises da tabela anexa referem-se a calcitas, calcáreos, calcáreos dolomíticos, dolomitas, hidromagnesita, anquerita e predazita.

Por terem sido efetuadas com finalidade técnica, não se fez uma análise completa, adotando-se o método resumido abaixo:

PERDA AO RUBRO - Determinada por calcinação em cadinho de porcelana, a cerca de $1000^{\circ} \mathrm{C}$, até constância de peso.

INSOLÚVEL EM HC1 - Determinado por dupla desidratação de 5 gramas de amostra finamente moída e secada em estufa a $110^{\circ} \mathrm{C}$, após ataque com HC1 1:1.
ÓXIDOS TOTAIS $\mathrm{R}^{2} \mathrm{O}^{3}$ - Determinados no filtrado do insolúvel, após oxidação com $\mathrm{HNO}^{3}$, por precipitação a quente, com $\mathrm{NH}^{4} \mathrm{OH}$.

ÓXIDO DE FERRO $\mathrm{Fe}^{2} \mathrm{O}^{3}$ - Determinado no total de $\mathrm{R}^{2} \mathrm{O}^{3}$, por titulação com $\mathrm{KMnO}^{4} \mathrm{n} / 10$.

ÓXIDO DE ALUMÍNIO $\mathrm{Al}^{2} \mathrm{O}^{3}$ - Diferença entre $\mathrm{R}^{2} \mathrm{O}^{3}$ e $\mathrm{Fe}^{2} \mathrm{O}^{3}$.

ÓXIDO DE MANGANÊS MnO - Determinado em parte alíquota correspondente a 1 grama de material, por titulação com $\mathrm{KMnO}^{4} \mathrm{n} / 10$ ou N/100, de acordo coma quantidade.

ÓXIDO DE CÁLCIO $\mathrm{CaO}$ - Determinado no filtrado de $\mathrm{R}^{2} \mathrm{O}^{3}$, por precipitação com oxalato de amônio; algumas vezes redissolvido e titulado com $\mathrm{KMnO}^{4} \mathrm{n} / 10$.

ÓXIDO DE MAGNÉSIO MgO - Nos filtrados da separação do cálcio, por precipitação com fosfato de sódio em meio amoniacal.

As análises sob números 67, 107, 116, 117, 176, 207, 269, 270, 272, 273, 328, 333, 337, 602, 603, 606, 630 e 658, referem-se a amostras de calcita. As sob os números 484 e 558, respectivamente a uma de anquerita e outra de hidromagnesita.

O insolúvel das amostras 844, 945, 442, 443, 816, $851,852,853$ e 907 , era constituído por silicato de magnésio; das amostras 1052, 1053, 1054 e 1055, por uma mistura de areia e mica, com pouca argila, predominando a primeira, e da amostra 1056, constituído, em quase sua totalidade, de argila.

As análises 277, 278, 279, 280, 281, 282, 283, 284 e 285, referem-se a nove médias feitas na jazida de Votuvurú. A análise 838 a uma amostra de predazita.

As restantes análises referem-se a calcáreos, calcáreos dolomíticos e dolomitas; é de ressaltar que, ao ser efetuado o ataque com $\mathrm{HC} 1$, afora exceções em calcáreos brancos, todos os outros demonstram possuir carbono grafítico, desprendendo concomitantemente odores de hidrocarburetos, com maior ou menor intensidade.

\section{RESUMO}

Apesar da natural existência de grande oscilação em todos os teores, em uma mesma jazida, das análises expostas na tabela anexa podemos concluir que:

1) as amostras com teor $\mathrm{MgO}$ até $3 \%$, representam $52,58 \%$ do total das análises; de 3 até 5, 7,62\%; de 5 até $10,6,77 \%$; e acima de $10,33,05 \%$.

2) com teor em insolúvel até $5 \%, 54,23 \%$; de 5 até 10, 16,10\%; e acima de 10, 27,96\%. 
3) das análises em que se fez a dosagem do $\mathrm{MnO}$, $47,27 \%$ deram resultado negativo.

4) com exceção da maior parte dos calcáreos brancos, todos os outros contém carbono grafítico.

5) os calcáreos da zona de Itaiacoca, Município de Ponta Grossa, além da mistura com silicato de magnésio, estão acompanhados de $\mathrm{Mg}(\mathrm{OH})^{2} \mathrm{em}$ teores variáveis.

6) apesar de algumas amostras de grandes jazidas apresentarem boa pureza, mister se torna um estudo rigoroso e completo das mesmas, de acordo com a finalidade, pois nelas existe grande variação, principalmente quanto ao $\mathrm{MgO}$.

7) em virtude de terem sido as análises feitas em material na sua maioria enviado a este Instituto, as porcentagens calculadas nos itens acima referemse somente às mesmas, não representando uma média no Estado. 
SÉRIE ASSUNGUí

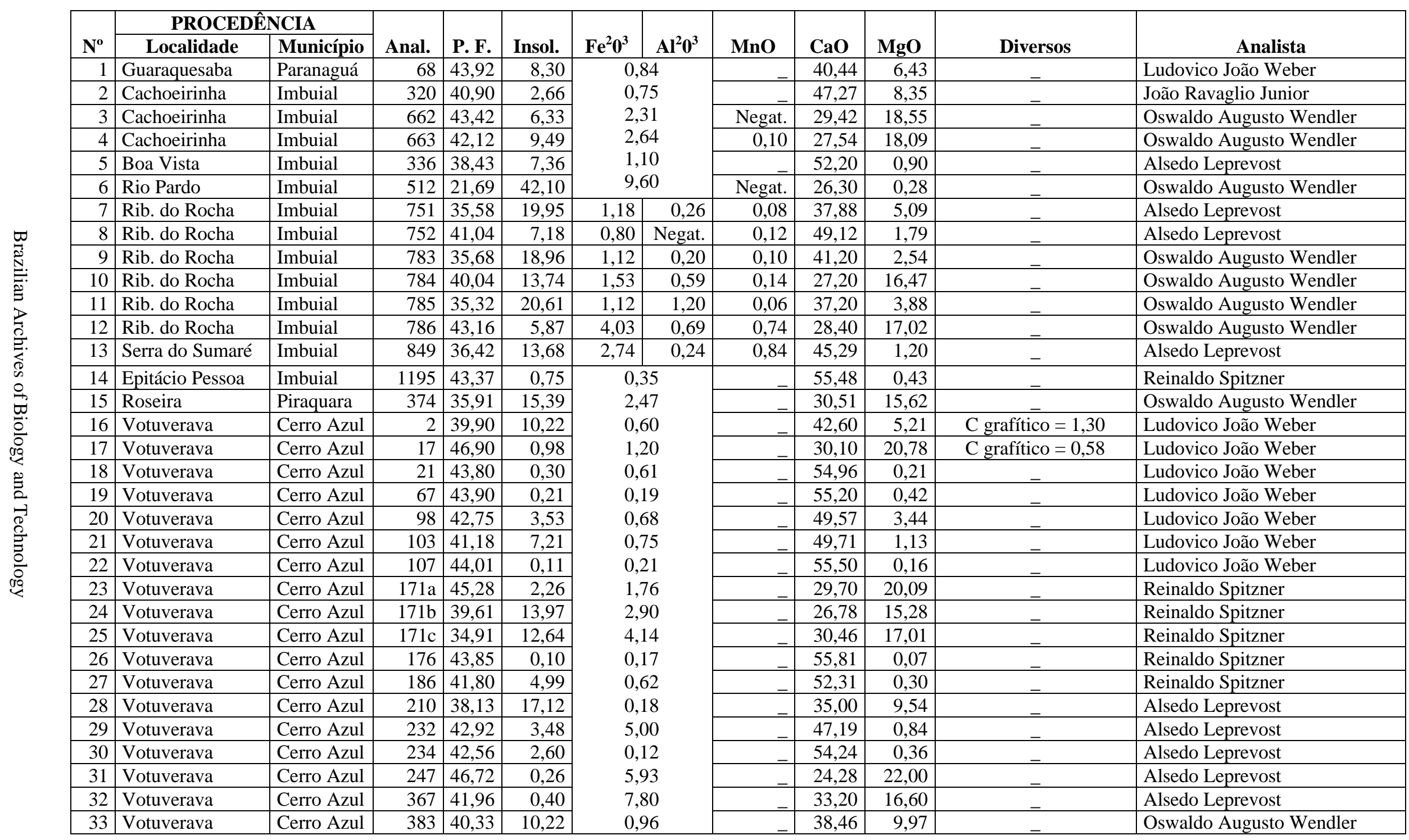




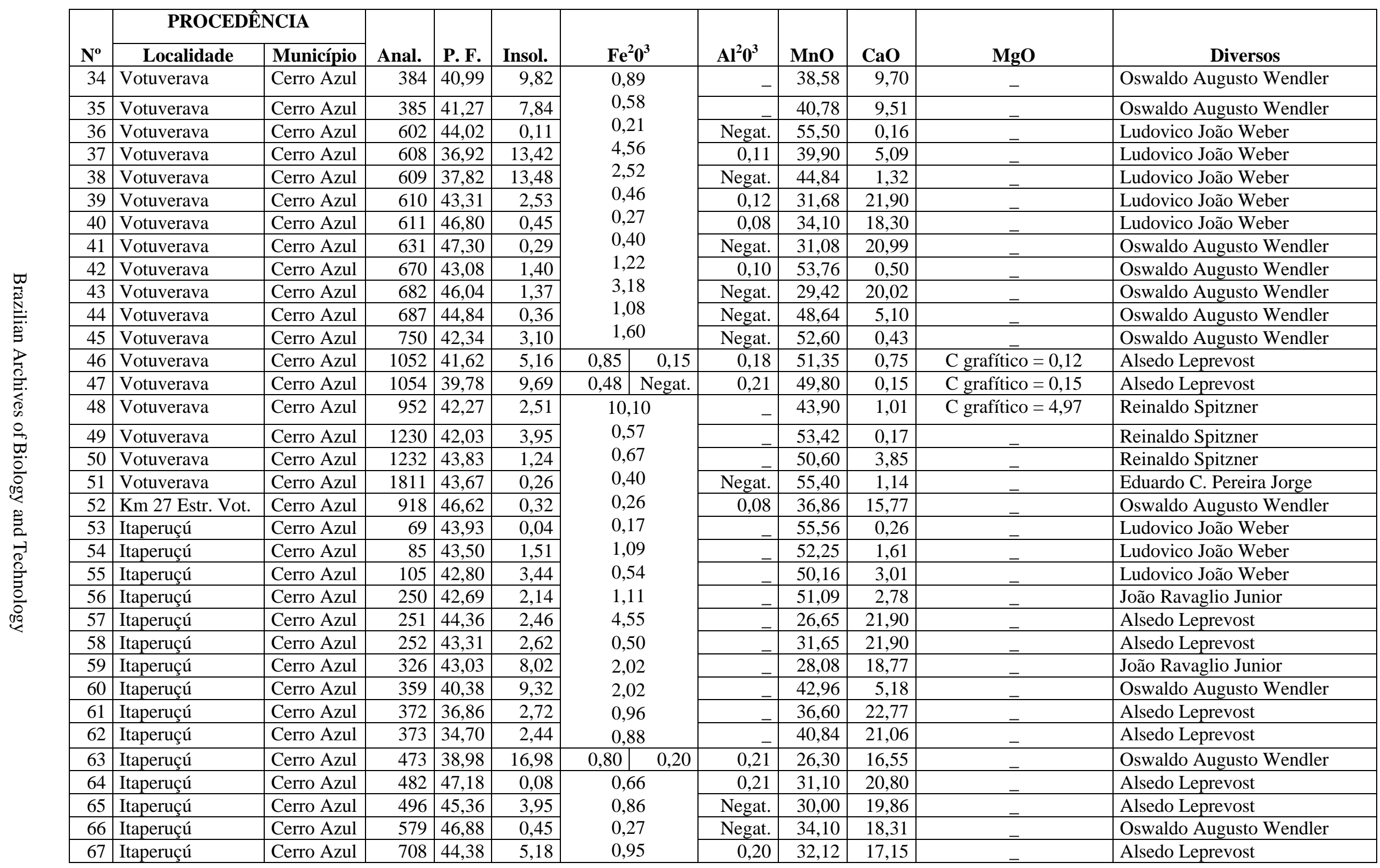




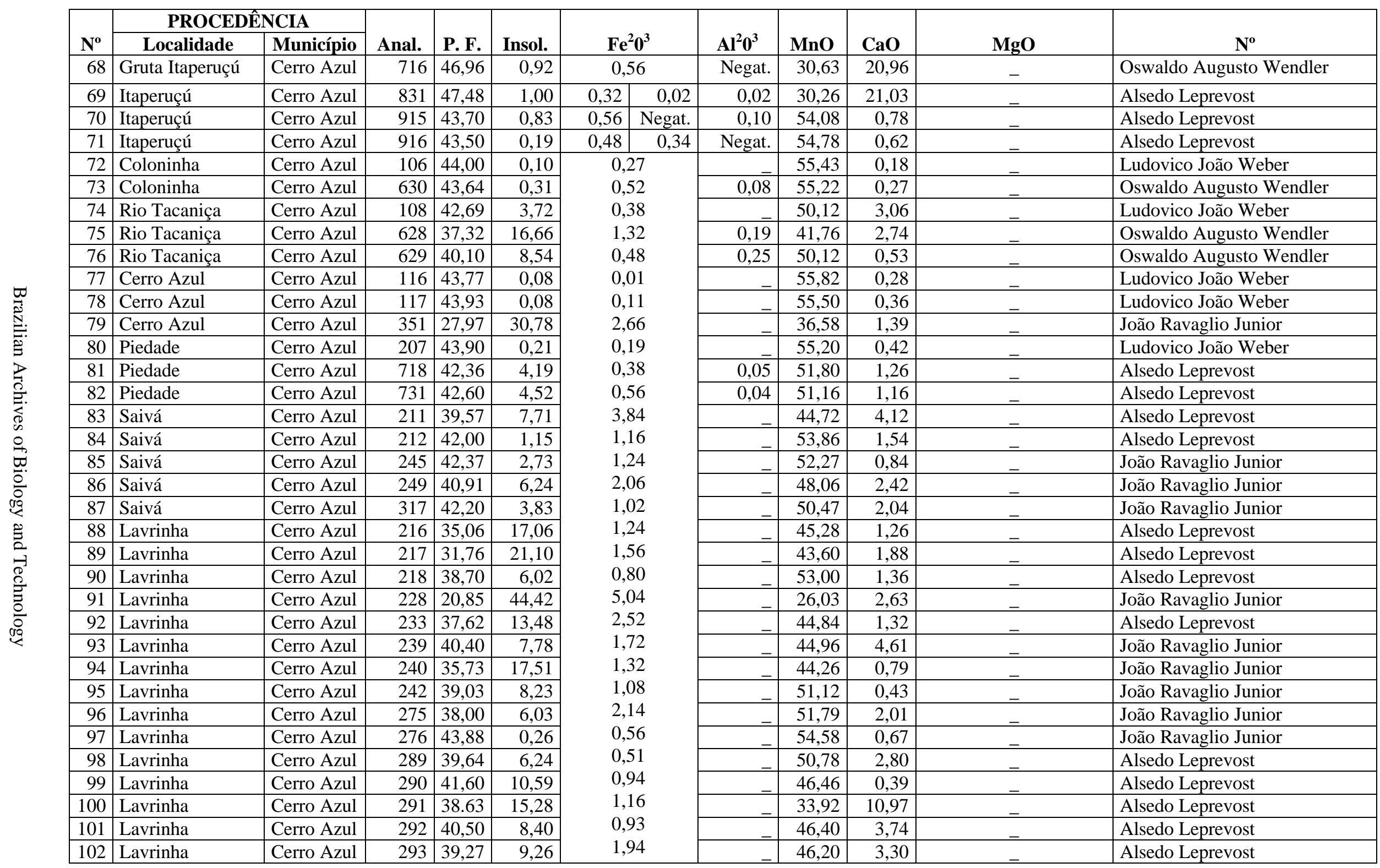




\begin{tabular}{|c|c|c|c|c|c|c|c|c|c|c|c|c|}
\hline \multirow[b]{2}{*}{$\mathbf{N}^{\mathbf{o}}$} & \multicolumn{2}{|c|}{ PROCEDÊNCIA } & \multirow[b]{2}{*}{ Anal. } & \multirow[b]{2}{*}{ P. F. } & \multirow[b]{2}{*}{ Insol. } & \multirow{2}{*}{\multicolumn{2}{|c|}{$\mathrm{Fe}^{2} \mathrm{O}^{3}$}} & \multirow[b]{2}{*}{$\mathbf{A l}^{2} \mathbf{0}^{3}$} & \multirow[b]{2}{*}{ MnO } & \multirow[b]{2}{*}{$\mathrm{CaO}$} & \multirow[b]{2}{*}{ MgO } & \multirow[b]{2}{*}{ Diversos } \\
\hline & Localidade & Município & & & & & & & & & & \\
\hline 103 & Lavrinha & Cerro Azul & 310 & 14,46 & 32,70 & \multirow{2}{*}{\multicolumn{2}{|c|}{$\begin{array}{c}17,40 \\
1,38\end{array}$}} & - & 33,60 & 1,75 & - & João Ravaglio Junior \\
\hline 104 & Lavrinha & Cerro Azul & 312 & 38,85 & 19,12 & & & & 36,30 & 4,32 & & João Ravaglio Junior \\
\hline 105 & Lavrinha & Cerro Azul & 316 & 40,68 & 7,11 & \multicolumn{2}{|c|}{0,89} & & 49,91 & 1,05 & - & João Ravaglio Junior \\
\hline 106 & Lavrinha & Cerro Azul & 335 & 37,23 & 12,06 & \multirow{2}{*}{\multicolumn{2}{|c|}{$\begin{array}{l}1,96 \\
1,46\end{array}$}} & & 47,96 & 0,74 & - & Alsedo Leprevost \\
\hline 107 & Lavrinha & Cerro Azul & 823 & 41,32 & 5,43 & & & Traços & 50,40 & 1,45 & - & Oswaldo Augusto Wendler \\
\hline 108 & Campinhos & Cerro Azul & 230 & 43,53 & 0,12 & \multicolumn{2}{|c|}{0,32} & & 53,80 & 1,19 & _. & João Ravaglio Junior \\
\hline 109 & Campinhos & Cerro Azul & 244 & 43,25 & 0,12 & \multirow{2}{*}{\multicolumn{2}{|c|}{$\begin{array}{l}0,80 \\
0,35\end{array}$}} & & 55,04 & 0,17 & & João Ravaglio Junior \\
\hline 110 & Campinhos & Cerro Azul & 297 & 43,44 & 0,05 & & & & 54,39 & 1,75 & - & Alsedo Leprevost \\
\hline 111 & Campinhos & Cerro Azul & 485 & 43,63 & 0,06 & \multicolumn{2}{|c|}{0,24} & Negat. & 53,84 & 2,25 & & Alsedo Leprevost \\
\hline 112 & Campinhos & Cerro Azul & 558 & 55,62 & 1,06 & \multirow{2}{*}{\multicolumn{2}{|c|}{$\begin{array}{l}0,64 \\
1,68\end{array}$}} & Negat. & 1,40 & 41,30 & - & Oswaldo Augusto Wendler \\
\hline 113 & Campinhos & Cerro Azul & 559 & 43,95 & 0,18 & & & Negat. & 50,75 & 3,42 & - & Oswaldo Augusto Wendler \\
\hline 114 & Campinhos & Cerro Azul & 573 & 21,20 & 53,78 & \multicolumn{2}{|c|}{$\begin{array}{l}0,40 \\
260\end{array}$} & Negat. & 23,46 & 1,15 & & Oswaldo Augusto Wendler \\
\hline 115 & Campinhos & Cerro Azul & 574 & 43,38 & 0,16 & \multirow{2}{*}{\multicolumn{2}{|c|}{$\begin{array}{l}2,60 \\
2,60\end{array}$}} & Negat. & 53,14 & 0,72 & - & Oswaldo Augusto Wendler \\
\hline 116 & Campinhos & Cerro Azul & 576 & 43,08 & 0,26 & & & Negat. & 53,49 & 0,54 & & Oswaldo Augusto Wendler \\
\hline 117 & Campinhos & Cerro Azul & 577 & 46,11 & 1,50 & \multicolumn{2}{|c|}{2,20} & Negat. & 31,80 & 18,38 & - & Oswaldo Augusto Wendler \\
\hline 118 & Campinhos & Cerro Azul & 578 & 43,30 & 4,10 & \multirow{2}{*}{\multicolumn{2}{|c|}{$\begin{array}{l}4,10 \\
0,04\end{array}$}} & Negat. & 33,24 & 15,26 & - & Oswaldo Augusto Wendler \\
\hline 119 & Corriola & Cerro Azul & 269 & 43,38 & 0,05 & & & & 55,85 & 0,06 & - & João Ravaglio Junior \\
\hline 120 & Corriola & Cerro Azul & 270 & 43,28 & 0,24 & \multicolumn{2}{|c|}{0,13} & & 55,56 & 0,06 & & João Ravaglio Junior \\
\hline 121 & Corriola & Cerro Azul & 308 & 41,00 & 5,82 & \multirow{2}{*}{\multicolumn{2}{|c|}{$\begin{array}{l}0,72 \\
0,19\end{array}$}} & & 51,50 & 0,93 & & João Ravaglio Junior \\
\hline 122 & Corriola & Cerro Azul & 603 & 43,90 & 0,21 & & & $0, \overline{15}$ & 55,12 & 0,43 & - & Ludovico João Weber \\
\hline 123 & Corriola & Cerro Azul & 606 & 43,90 & 0,18 & 0 , & & Negat. & 55,40 & 0,38 & & Ludovico João Weber \\
\hline 124 & Corriola & Cerro Azul & 1047 & 43,68 & 1,18 & 0,20 & Negat. & 0,02 & 54,35 & 0,40 & C grafítico $=0,33$ & Alsedo Leprevost \\
\hline 125 & Corriola & Cerro Azul & 1053 & 43.62 & 0,72 & Traços & Traços & 0,03 & 55,30 & 0,19 & C grafítico $=0,23$ & Alsedo Leprevost \\
\hline 126 & Corriola & Cerro Azul & 1055 & 41,90 & 7,88 & 0,80 & 0,25 & Negat. & 39,20 & 10,10 & $\mathrm{C}$ grafítico $=0,15$ & Alsedo Leprevost \\
\hline 127 & Vutuvurú & Cerro Azul & 277 & 42,28 & 1,00 & 3 , & & & 51,89 & 1,15 & $\ldots$ & João Ravaglio Junior \\
\hline 128 & Vutuvurú & Cerro Azul & 278 & 42,83 & 2,44 & 1, & & & 48,52 & 5,15 & - & João Ravaglio Junior \\
\hline 129 & Vutuvurú & Cerro Azul & 279 & 40,40 & 5,62 & 1, & & & 50,57 & 1,47 & - & João Ravaglio Junior \\
\hline 130 & Vutuvurú & Cerro Azul & 280 & 41,56 & 4,96 & 0 & & & 51,06 & 1,68 & - & João Ravaglio Junior \\
\hline 131 & Vutuvurú & Cerro Azul & 281 & 42,16 & 3,55 & 0 & & & 52,00 & 1,36 & - & João Ravaglio Junior \\
\hline 132 & Vutuvurú & Cerro Azul & 282 & 42,84 & 2,27 & 0 & & & 52,67 & 1,45 & & João Ravaglio Junior \\
\hline 133 & Vutuvurú & Cerro Azul & 283 & 41,97 & 2,38 & 1, & & & 53,39 & 0,87 & - & João Ravaglio Junior \\
\hline 134 & Vutuvurú & Cerro Azul & 284 & 42,18 & 2,48 & 0 & & & 53,74 & 1,02 & $=$ & João Ravaglio Junior \\
\hline 135 & Vutuvurú & Cerro Azul & 285 & 34,97 & 1,86 & 1, & & & 52,89 & 8,76 & & João Ravaglio Junior \\
\hline 136 & Vutuvurú & Cerro Azul & 298 & 42,61 & 1,34 & 0 & & & 53,50 & 2,02 & - & Alsedo Leprevost \\
\hline 137 & Madre & Cerro Azul & 296 & 37,42 & 11,98 & 0 & & & 49,29 & 0,56 & & Alsedo Leprevost \\
\hline
\end{tabular}




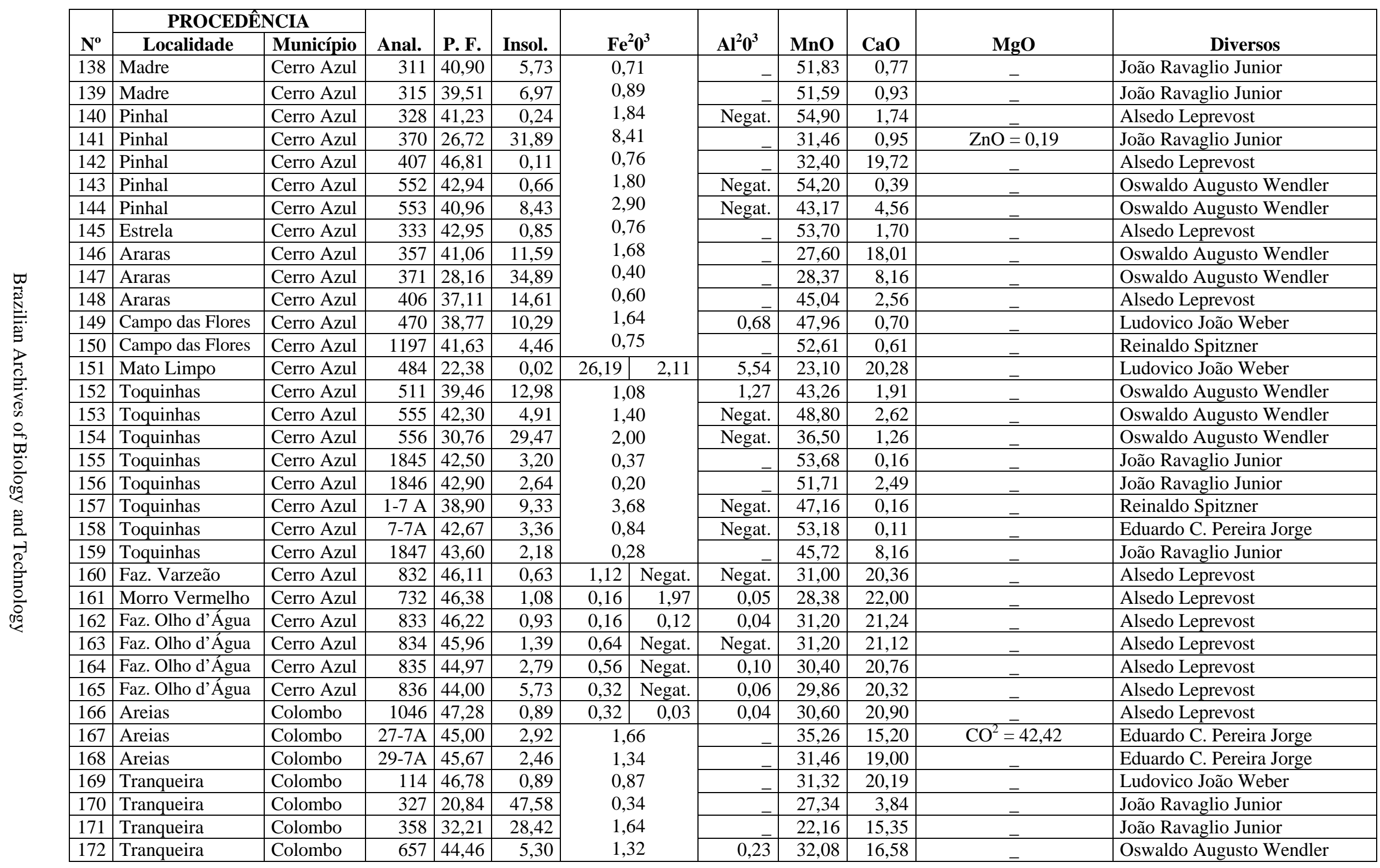




\begin{tabular}{|c|c|c|c|c|c|c|c|c|c|c|c|c|}
\hline \multirow[b]{2}{*}{$\mathbf{N}^{\mathbf{o}}$} & \multicolumn{2}{|c|}{ PROCEDÊNCIA } & \multirow[b]{2}{*}{ Anal. } & \multirow[b]{2}{*}{ P. F. } & \multirow[b]{2}{*}{ Insol. } & \multirow{2}{*}{\multicolumn{2}{|c|}{$\mathrm{Fe}^{2} \mathbf{0}^{3}$}} & \multirow[b]{2}{*}{$\mathbf{A l}^{2} \mathbf{0}^{3}$} & \multirow[b]{2}{*}{ MnO } & \multirow[b]{2}{*}{$\mathrm{CaO}$} & \multirow[b]{2}{*}{ MgO } & \multirow[b]{2}{*}{ Diversos } \\
\hline & Localidade & Município & & & & & & & & & & \\
\hline 173 & Tranqueira & Colombo & 780 & 46,63 & 1,54 & \multicolumn{2}{|c|}{0,34} & 0,02 & 31,54 & 19,97 & - & Oswaldo Augusto Wendler \\
\hline 174 & Tranqueira & Colombo & 850 & 46,58 & 0,02 & 0,16 & 0,06 & 0,10 & 32,18 & 21,28 & & Alsedo Leprevost \\
\hline 175 & Tranqueira & Colombo & 917 & 46,80 & 0,48 & 0,28 & 0,06 & Negat. & 32,12 & 20,14 & - & Alsedo Leprevost \\
\hline 176 & Tranqueira & Colombo & 1010 & 46,55 & 1,33 & 0,28 & 0,51 & Negat. & 31,96 & 19,40 & - & Alsedo Leprevost \\
\hline 177 & Tranqueira & Colombo & 1877 & 35,92 & 20,18 & \multirow{2}{*}{\multicolumn{2}{|c|}{$\begin{array}{l}1,48 \\
0,42\end{array}$}} & & 25,52 & 15,24 & _ & Eduardo C. Pereira Jorge \\
\hline 178 & Bacaetava & Colombo & 213 & 43,49 & 0,25 & & & & 55,63 & 0,16 & - & João Ravaglio Junior \\
\hline 179 & Bacaetava & Colombo & 214 & 43,53 & 0,91 & \multirow{2}{*}{\multicolumn{2}{|c|}{$\begin{array}{l}0,66 \\
0,28\end{array}$}} & & 53,79 & 0,97 & & João Ravaglio Junior \\
\hline 180 & Bacaetava & Colombo & 229 & 43,80 & 0,09 & & & & 53,74 & 0,86 & - & João Ravaglio Junior \\
\hline 181 & Bacaetava & Colombo & 253 & 42,23 & 8,04 & \multicolumn{2}{|c|}{9,90} & & 25,95 & 13,50 & _- & Alsedo Leprevost \\
\hline 182 & Bacaetava & Colombo & 294 & 46,43 & 0,26 & \multirow{2}{*}{\multicolumn{2}{|c|}{$\begin{array}{l}1,50 \\
2,91\end{array}$}} & & 33,47 & 18,30 & & Alsedo Leprevost \\
\hline 183 & Bacaetava & Colombo & 295 & 42,60 & 018 & & & & 52,04 & 2,26 & - & Alsedo Leprevost \\
\hline 184 & Bacaetava & Colombo & 337 & 43,13 & 1,22 & \multicolumn{2}{|c|}{1,22} & & 53,98 & 0,40 & & João Ravaglio Junior \\
\hline 185 & Bacaetava & Colombo & 350 & 29,25 & 25,83 & \multirow{4}{*}{\multicolumn{2}{|c|}{$\begin{array}{c}10,01 \\
1,38 \\
2,82 \\
1,80\end{array}$}} & & 33,49 & 1,23 & - & João Ravaglio Junior \\
\hline 186 & Bacaetava & Colombo & 390 & 33,07 & 5,87 & & & & 40,44 & 19,19 & - & Oswaldo Augusto Wendler \\
\hline 187 & Bacaetava & Colombo & 391 & 38,11 & 12,33 & & & & 27,59 & 19,12 & - & Oswaldo Augusto Wendler \\
\hline 188 & Bacaetava & Colombo & 416 & 45,79 & 5,08 & & & & 36,79 & 10,50 & - & Oswaldo Augusto Wendler \\
\hline 189 & Bacaetava & Colombo & 441 & 43,20 & 3,26 & 1,20 & 0,40 & $0, \overline{01}$ & 51,10 & 0,79 & & Oswaldo Augusto Wendler \\
\hline 190 & Bacaetava & Colombo & 478 & 42,50 & 3,58 & \multirow{2}{*}{\multicolumn{2}{|c|}{$\begin{array}{l}6,02 \\
280\end{array}$}} & Negat. & 35,20 & 12,72 & - & Oswaldo Augusto Wendler \\
\hline 191 & Bacaetava & Colombo & 481 & 43,06 & 3,88 & & & 1,07 & 48,20 & 1,01 & - & Oswaldo Augusto Wendler \\
\hline 192 & Bacaetava & Colombo & 483 & 43,96 & 0,06 & \multicolumn{2}{|c|}{0,18} & Negat. & 55,02 & 0,76 & & Alsedo Leprevost \\
\hline 193 & Bacaetava & Colombo & 551 & 43,06 & 0,54 & \multirow{5}{*}{\multicolumn{2}{|c|}{$\begin{array}{l}1,50 \\
1,28 \\
0,12 \\
0,05 \\
0,67\end{array}$}} & Negat. & 53,30 & 1,59 & - & Oswaldo Augusto Wendler \\
\hline 194 & Tamandaré & Colombo & 271 & 37,50 & 12,80 & & & & 45,01 & 3,31 & & João Ravaglio Junior \\
\hline 195 & Tamandaré & Colombo & 272 & 43,30 & 0,13 & & & & 55,68 & 0,07 & - & João Ravaglio Junior \\
\hline 196 & Tamandaré & Colombo & 273 & 43,17 & 2,19 & & & & 54,28 & 0,12 & & João Ravaglio Junior \\
\hline 197 & Capivarí & Colombo & 526 & 45,90 & 2,96 & & & Negat. & 30,30 & 20,15 & & Oswaldo Augusto Wendler \\
\hline 198 & Capivarí & Colombo & 545 & 21,44 & 50,78 & 0,40 & 0,44 & 0,85 & 23,44 & 2,67 & _- & Oswaldo Augusto Wendler \\
\hline 199 & Poço Negro & Colombo & 527 & 42,85 & 3,43 & \multicolumn{2}{|c|}{0,86} & Negat. & 50,03 & 2,82 & & Oswaldo Augusto Wendler \\
\hline 200 & São Gabriel & Colombo & 900 & 46,22 & 2,22 & 0,32 & 1,74 & 0,10 & 30,90 & 18,60 & - & Oswaldo Augusto Wendler \\
\hline 201 & Bateias & Campo Largo & 446 & 39,73 & 43,00 & 0,80 & 2,20 & 1,32 & 10,20 & 2,67 & - & Oswaldo Augusto Wendler \\
\hline 202 & Bateias & Campo Largo & 447 & 41,08 & 10,12 & 1,60 & Negat. & & 33,20 & 13,97 & & Oswaldo Augusto Wendler \\
\hline 203 & Bateias & Campo Largo & 476 & 40,12 & 9,04 & \multirow{5}{*}{\multicolumn{2}{|c|}{$\begin{array}{l}5,80 \\
5,60 \\
2,30 \\
3,92 \\
0,82\end{array}$}} & $0, \overline{\overline{9}}$ & 42,90 & 1,32 & - & Oswaldo Augusto Wendler \\
\hline 204 & Bateias & Campo Largo & 477 & 19,94 & 52,72 & & & Negat. & 20,60 & 1,12 & & Oswaldo Augusto Wendler \\
\hline 205 & Bateias & Campo Largo & 480 & 38,49 & 11,10 & & & Negat. & 46,20 & 1,88 & & Oswaldo Augusto Wendler \\
\hline 206 & Bateias & Campo Largo & 488 & 22,88 & 34,04 & & & Negat. & 23,52 & 13,38 & $\mathrm{BaO}=1,47 \mathrm{SO}^{3}=0,76$ & Oswaldo Augusto Wendler \\
\hline 207 & Bateias & Campo Largo & 493 & 38,83 & 12,29 & & & 0,46 & 43,36 & 4,22 & & Alsedo Leprevost \\
\hline
\end{tabular}




\begin{tabular}{|c|c|c|c|c|c|c|c|c|c|c|c|c|}
\hline & PROCEDÊ & NCIA & & & & & & & & & & \\
\hline $\mathbf{N}^{\mathbf{o}}$ & Localidade & Município & Anal. & P. F. & Insol. & \multicolumn{2}{|c|}{$\mathrm{Fe}^{2} \mathbf{0}^{3}$} & $\mathrm{Al}^{2} \mathbf{0}^{3}$ & MnO & $\mathrm{CaO}$ & MgO & Diversos \\
\hline 208 & Bateias & Campo Largo & 494 & 38,67 & 11,78 & \multicolumn{2}{|c|}{2,28} & Negat. & 42,72 & 4,52 & - & Alsedo Leprevost \\
\hline 209 & Bateias & Campo Largo & 495 & 37,01 & 17,62 & \multirow{5}{*}{\multicolumn{2}{|c|}{$\begin{array}{l}1,14 \\
1,50 \\
6,00 \\
0,62 \\
0,75\end{array}$}} & 0,51 & 40,64 & 3,10 & & Alsedo Leprevost \\
\hline 210 & Bateias & Campo Largo & 1031 & 36,58 & 22,70 & & & Negat. & 21,94 & 17,40 & & Alsedo Leprevost \\
\hline 211 & Morro do Cal & Campo Largo & 475 & 44,03 & 3,52 & & & Negat. & 30,00 & 16,43 & & Oswaldo Augusto Wendler \\
\hline 212 & Morro do Cal & Campo Largo & 658 & 43,82 & 0,33 & & & Negat. & 55,04 & 0,21 & _- & Oswaldo Augusto Wendler \\
\hline 213 & Morro do Cal & Campo Largo & 661 & 42,94 & 2,28 & & & Negat. & 52,76 & 1,26 & & Oswaldo Augusto Wendler \\
\hline 214 & Ouro Fino & Campo Largo & 503 & 29,26 & 19,42 & 2,41 & 0,19 & Negat. & 30,50 & 18,26 & & Oswaldo Augusto Wendler \\
\hline 215 & Ouro Fino & Campo Largo & 595 & 44,72 & 5,01 & \multirow{2}{*}{\multicolumn{2}{|c|}{$\begin{array}{l}0,86 \\
2,16\end{array}$}} & 0,10 & 29,82 & 19,55 & - & Oswaldo Augusto Wendler \\
\hline 216 & São Luis & Campo Largo & 664 & 45,83 & 1,82 & & & Negat. & 32,18 & 18,03 & & Oswaldo Augusto Wendler \\
\hline 217 & Est. Cerne Km. 27 & Campo Largo & 1032 & 37,60 & 16,33 & \multirow{2}{*}{\multicolumn{2}{|c|}{$\begin{array}{l}0,90 \\
0,62\end{array}$}} & Traços & 42,95 & 2,30 & & Alsedo Leprevost \\
\hline 218 & Morros & Castro & 109 & 44,40 & 1,20 & & & & 46,74 & 7,02 & & Ludovico João Weber \\
\hline 219 & Morros & Castro & 508 & 37,42 & 18,03 & \multirow{2}{*}{\multicolumn{2}{|c|}{$\begin{array}{l}2,20 \\
1,60\end{array}$}} & $0,0 \overline{7}$ & 31,41 & 10,79 & & Ludovico João Weber \\
\hline 220 & \begin{tabular}{|l|} 
Itaicoca \\
\end{tabular} & Ponta Grossa & 215 & 41,73 & 1,63 & & & & 53,10 & 1,92 & & Alsedo Leprevost \\
\hline 221 & Itaicoca & Ponta Grossa & 838 & 39,22 & 0,10 & 0,08 & 0,30 & 0,07 & 35,96 & 24,40 & $\mathrm{H}^{2} \mathrm{O}=10,91 ; \mathrm{CO}^{2}=28,31$ & Alsedo Leprevost \\
\hline 222 & Itaicoca & Ponta Grossa & 844 & 14,48 & 46,38 & 0,80 & 1,35 & 0,70 & 13,65 & 22,80 & $\mathrm{H}^{2} \mathrm{O}=3,18 ; \mathrm{CO}^{2}=11,30$ & Alsedo Leprevost \\
\hline 223 & Itaicoca & Ponta Grossa & 945 & 18,70 & 39,17 & Taços & 0,40 & Traços & 20,80 & 20,90 & - & Alsedo Leprevost \\
\hline 224 & Itaicoca & Ponta Grossa & 1044 & 47,26 & 0,50 & 0,80 & Negat. & Negat. & 30,85 & 20,70 & - & Alsedo Leprevost \\
\hline 225 & Biscaia & Ponta Grossa & 442 & 26,94 & 42,85 & 0,40 & 0,50 & 0,52 & 16,40 & 12,38 & $=$ & Oswaldo Augusto Wendler \\
\hline 226 & Anta Moura & Ponta Grossa & 443 & 13,08 & 70,32 & \multicolumn{2}{|c|}{0,20} & & 14,00 & 0,36 & - & Oswaldo Augusto Wendler \\
\hline 227 & Anta Moura & Ponta Grossa & 816 & 16,76 & 62,01 & 0,20 & 0,60 & $0, \overline{10}$ & 19,38 & 0,29 & & Oswaldo Augusto Wendler \\
\hline 228 & Anta Moura & Ponta Grossa & 851 & 24,33 & 28,46 & \multirow{3}{*}{\multicolumn{2}{|c|}{$\begin{array}{l}1,00 \\
0,90 \\
1,20\end{array}$}} & Traços & 31,85 & 14,40 & $\mathrm{H}^{2} \mathrm{O}=0,58 ; \mathrm{CO}^{2}=23,75$ & Alsedo Leprevost \\
\hline 229 & Anta Moura & Ponta Grossa & 852 & 27,28 & 25,30 & & & Traços & 31,00 & 15,60 & $\mathrm{H}^{2} \mathrm{O}=2,64 ; \mathrm{CO}^{2}=25,30$ & Alsedo Leprevost \\
\hline 230 & Anta Moura & Ponta Grossa & 853 & 29,14 & 22,60 & & & Traços & 34,10 & 12,90 & $\mathrm{H}^{2} \mathrm{O}=3,52 ; \mathrm{CO}^{2}=25,62$ & Alsedo Leprevost \\
\hline 231 & Anta Moura & Ponta Grossa & 907 & 10,04 & 56,54 & 0,40 & 0,10 & Negat. & 8,10 & 24,46 & & Oswaldo Augusto Wendler \\
\hline
\end{tabular}




\section{SÉRIE PASSA DOIS}

Fácies Terezina

\begin{tabular}{|c|c|c|c|c|c|c|c|c|c|c|c|c|}
\hline \multirow[b]{2}{*}{$\mathbf{N}^{\mathbf{o}}$} & \multicolumn{2}{|c|}{ PROCEDÊNCIA } & \multirow[b]{2}{*}{ Anal. } & \multirow[b]{2}{*}{ P. F. } & \multirow[b]{2}{*}{ Insol. } & \multirow[b]{2}{*}{$\mathrm{Fe}^{2} \mathbf{0}^{3}$} & \multirow[b]{2}{*}{$\mathrm{Al}^{2} \mathbf{0}^{3}$} & \multirow[b]{2}{*}{ MnO } & \multirow[b]{2}{*}{$\mathrm{CaO}$} & \multirow[b]{2}{*}{ MgO } & \multirow[b]{2}{*}{ Diversos } & \multirow[b]{2}{*}{ Analista } \\
\hline & Localidade & Município & & & & & & & & & & \\
\hline 232 & Braço do Potinga & Mallet & 104 & 39,32 & 11,26 & & & - & 48,04 & 0,62 & - & Ludovico João Weber \\
\hline
\end{tabular}

SÉRIE ITARARÉ-TUBARÃO

\begin{tabular}{|c|c|c|c|c|c|c|c|c|c|c|c|c|}
\hline \multirow[b]{2}{*}{$\mathbf{N}^{\mathbf{o}}$} & \multicolumn{2}{|c|}{ PROCEDÊNCIA } & \multirow[b]{2}{*}{ Anal. } & \multirow[b]{2}{*}{ P. F. } & \multirow[b]{2}{*}{ Insol. } & \multirow[b]{2}{*}{$\mathrm{Fe}^{2} \mathbf{0}^{3}$} & \multirow[b]{2}{*}{$\mathbf{A l}^{2} \mathbf{0}^{3}$} & \multirow[b]{2}{*}{ MnO } & \multirow[b]{2}{*}{$\mathrm{CaO}$} & \multirow[b]{2}{*}{ MgO } & \multirow[b]{2}{*}{ Diversos } & \multirow[b]{2}{*}{ Analista } \\
\hline & Localidade & Município & & & & & & & & & & \\
\hline 233 & Tomazina & Tomazina & $155 \mathrm{a}$ & 38,38 & 7,60 & \multirow{3}{*}{\multicolumn{2}{|c|}{$\begin{array}{l}2,90 \\
2,70 \\
4,40\end{array}$}} & & 48,92 & 1,96 & - & Reinaldo Spitzner \\
\hline 234 & Tomazina & Tomazina & $155 b$ & 35,80 & 16,00 & & & & 45,05 & 1,06 & - & Reinaldo Spitzner \\
\hline 235 & Tomazina & Tomazina & 259 & 36,72 & 12,58 & & & & 45,20 & 1,05 & & Alsedo Leprevost \\
\hline 236 & Tomazina & Tomazina & 1056 & 34,60 & 21,81 & 1,20 & 0,20 & Negat. & 42,15 & 0,07 & C grafítico $=0,09$ & Alsedo Leprevost \\
\hline
\end{tabular}

